\title{
Real Time Control System for the National Spherical Torus Experiment (NSTX)
}

\author{
C. Neumeyer, D. Gates, T. Gibney, R. Hatcher, S. Kaye, \\ R. Marsala, D. Mueller, G. Oliaro, S. Ramakrishnan \\ Princeton University Plasma Physics Laboratory* \\ Princeton, New Jersey 08543
}

\begin{abstract}
The NSTX [1] is a national facility for the study of plasma confinement, heating, and current drive in a low aspect ratio, spherical torus (ST) configuration. The ST configuration is an alternate magnetic confinement concept which is characterized by high $\beta$ (ratio plasma pressure to magnetic field pressure) and low toroidal field compared to conventional tokamaks, and could provide a pathway to the realization of a practical fusion power source.

The NSTX depends on a real time, high speed, synchronous, and deterministic control system acting on a system of thyristor rectifier power supplies to 1) establish the initial magnetic field configuration; 2) initiate plasma within the vacuum vessel; 3) inductively drive plasma current; and 4) control plasma position and shape. For the initial "day 0" 1st plasma operations (Feb. 1999), the system was limited to closed loop proportional-integral current control of the power supplies based on preprogrammed reference waveforms. For the next "day 1" phase of operations beginning mid-summer 1999 the loop will be closed on plasma current and position. The ultimate "day $2^{\prime \prime}$ system is envisioned to include real time reconstruction of the plasma internal current distribution so that control can be exercised over internal plasma parameters such as current and pressure profile.
\end{abstract}

This paper addresses the day 1 system, with emphasis on the magnet power supply control. Companion papers address plasma control $[2,3]$.

\section{INTRODUCTION}

A cross section of the NSTX device is shown in Figure 1. Parameters and ratings are summarized in Table 1. A hydrogen (deuterium) plasma is contained in an axi-symmetric toroidal vacuum chamber surrounded by water cooled copper magnets which carry current in the toroidal and poloidal directions to produce poloidal field (PF) and toroidal field (TF), respectively. The plasma current Ip flows in the toroidal direction. The net magnetic field is predominant in the toroidal direction but with a helical twist due to the poloidal field from the PF coils and plasma. Plasma particles tend to follow the magnetic field lines and are thereby confined. The vertical component of the magnetic field in the $\mathrm{z}$ direction $(\mathrm{Bz})$ exerts radial $\mathrm{J} \times \mathrm{B}$ force on the plasma particles and the radial component $B_{r} a$ horizontal force. The plasma cross sectional shape and current profile result from an equilibrium force balance between the $\mathbf{J}$ $\mathrm{x} \mathrm{B}$ forces arising from the applied fields and the plasma current, along with its kinetic gas pressure.

\footnotetext{
${ }^{*}$ Under USDOE Contract \#DE-AC02-76-CHO3073
}

\begin{tabular}{|l|l|l|}
\hline Major Radius $\left(\mathrm{R}_{\mathbf{0}}\right)$ & $\mathbf{8 5 . 4}$ & $\mathrm{cm}$ \\
\hline Aspect Ratio (R/a) & 1.25 & - \\
\hline Plasma Current & 1.0 & $\mathrm{MA}$ \\
\hline Toroidal Field @ $\mathrm{R}_{\mathbf{0}}$ (max.) & 6.0 & $\mathrm{kG}$ \\
\hline Plasma Ramp Time & $0.2-0.4$ & $\mathrm{sec}$ \\
\hline Plasma Flat Top (max.) & 5.0 & $\mathrm{sec}$ \\
\hline Repetition Period (min.) & 300.0 & $\mathrm{sec}$ \\
\hline
\end{tabular}

Table 1

NSTX Parameters and Ratings

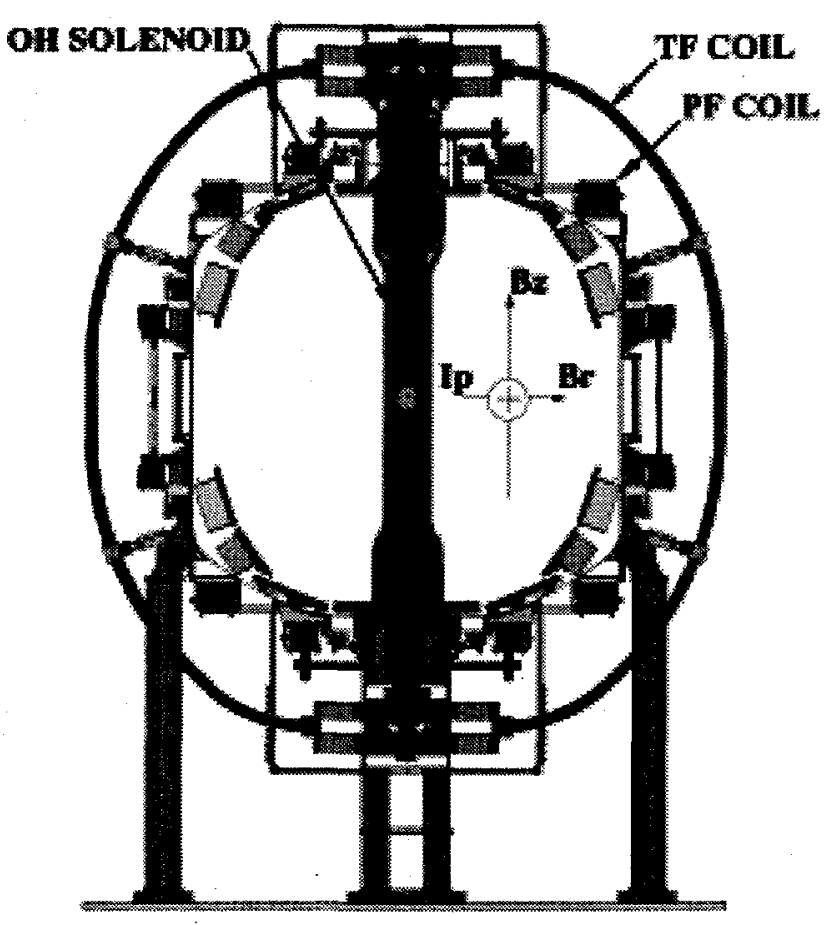

Figure 1: Cross Section View of the NSTX Device 


\section{DISCLAIMER}

This report was prepared as an account of work sponsored by an agency of the United States Government. Neither the United States Government nor any agency thereof, nor any of their employees, make any warranty, express or implied, or assumes any legal liability or responsibility for the accuracy, completeness, or usefulness of any information, apparatus, product, or process disclosed, or represents that its use would not infringe privately owned rights. Reference herein to any specific commercial product, process, or service by trade name, trademark, manufacturer, or otherwise does not necessarily constitute or imply its endorsement, recommendation, or favoring by the United States Government or any agency thereof. The views and opinions of authors expressed herein do not necessarily state or reflect those of the United States Government or any agency thereof. 


\section{DISCLAIMER}

Portions of this document may be illegible in electronic image products. Images are produced from the best available original document. 
During a pulse the typical sequence of events is as follows:

- Central Ohmic Heating $(\mathrm{OH})$ solenoid coil is precharged

- TF is applied

- Fuel gas is injected

- OH coil current is rapidly decreased, such that toroidal loop voltage is generated to ionize the fuel gas

- Plasma current is driven by induction by $\mathrm{OH}$ coil

- PF coils are used to control plasma shape and position

A conceptual block diagram of the system is given in Figure 2. Typically, upon initiation of a pulse the Power Supply Real Time Controller (PSRTC) performs closed loop current control to precharge the $\mathrm{OH}$ coil, initiate the TF coil current, and pre-bias other PF coils as required. Then, at the start of a pre-programmed time window, the Plasma Real Time Controller $[1,2]$ initiates the plasma and controls plasma current, position, shape, etc. via voltage requests to the PSRTC. Upon termination of the plasma current the PSRTC extinguishes the current in all coil systems according to preprogrammed current derivatives.

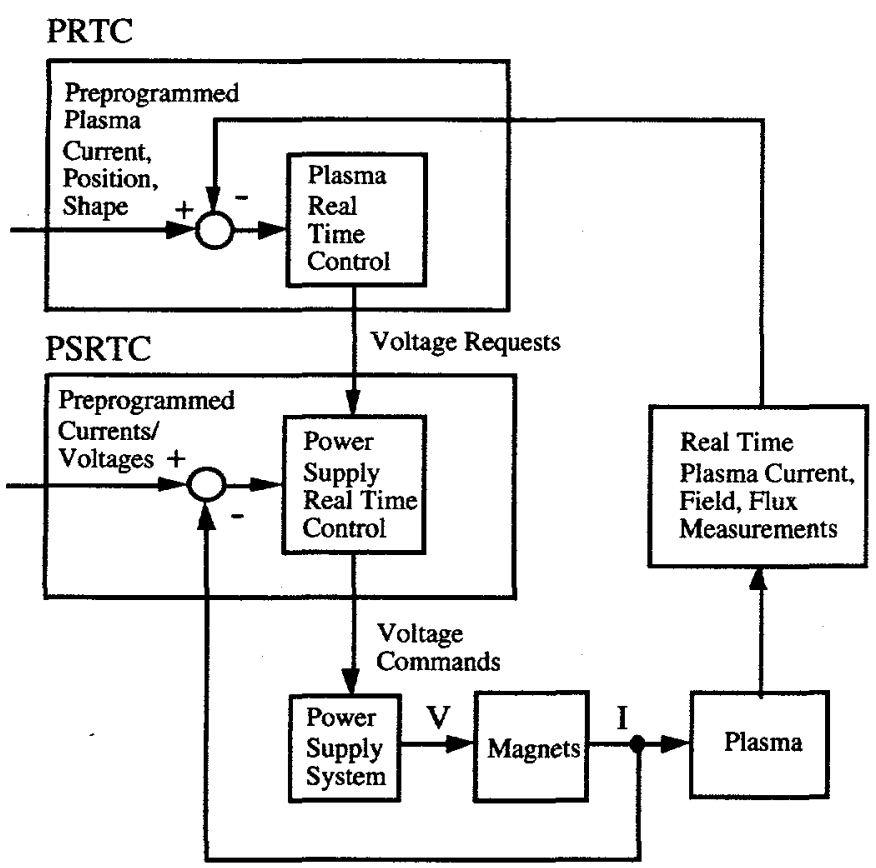

Figure 2: Conceptual Block Diagram of Control System

\section{POWER SuPPlies \& CiRCUITS TO BE CONTROLLED}

\section{A. Power Supplies}

The PPPL "Power Supplies" (PS), consist of thirty nine (39) modular 12-pulse phase controlled thyristor rectifiers. Each PS consists of two 6-pulse "Power Supply. Sections" (PSS) which are electrically isolated from each other but subject to the same "Firing Generator" (FG). Each PSS is equipped with a full rated bypass module which can carry the load current when the rectifiers are suppressed. Each PSS is rated to produce a maximum average DC output voltage of $1012.85 \mathrm{VDC}(\approx 1 \mathrm{kV})$, no load, with $13.8 \mathrm{kV}$ AC input voltage applied to the converter transformer primary. Nominal pulse current rating of each PSS is $24 \mathrm{kA}-6 \mathrm{sec} / 300 \mathrm{sec}$. The FG of each PS controls, in effect, the voltage produced by its two PSS by phase controlling the thyristor firing pulses in accordance with a Control Word received as an input from the PSRTC. The Control Word is an 11 bit digital command.

\section{B. Circuits}

Circuit types to be controlled are as depicted in Figure 3, where the boxed thyristor symbol represents one or more PSS connected in series/parallel combination. The lines connecting the circles represent removable bus links.
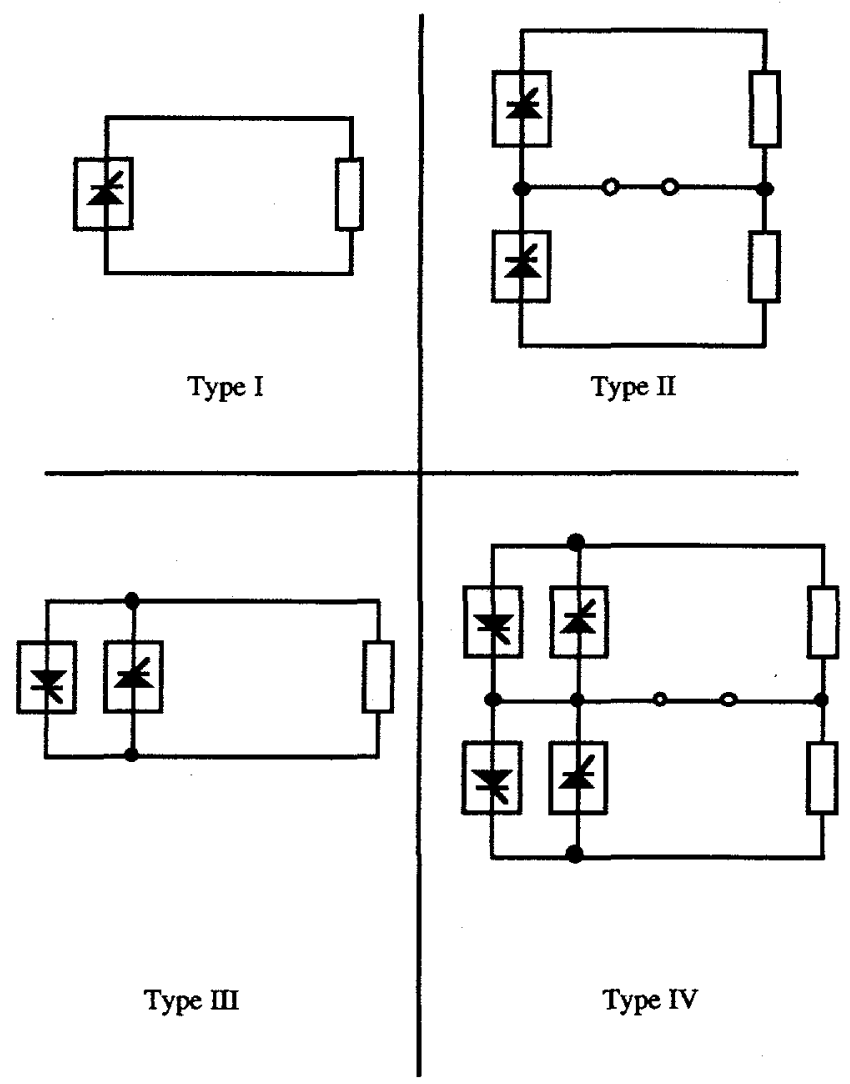

Figure 3: NSTX Circuit Configurations

a. Type I is a "Unipolar", "2-Wire" circuit. Current flows in one direction through one load coil group.

b. Type II is a "Unipolar", "3-Wire" circuit. Current flows in one direction through two load coil groups; the magnitude of the current may be different in the two "twin" load coil groups.

c. Type III is a "Bipolar", "2-Wire" circuit. Current flows in either direction through one load coil group.

d. Type IV is a "Bipolar", "3-Wire" circuit. Current flows in either direction through two load coil groups; the magnitude and polarity of the current may be different in the two "twin" load coil groups. 
A listing of the NSTX circuits along with type, number of DC poles, and number of PSS is given in Table 2.

Table 2

NSTX Circuit Types, Poles, and PSS

\begin{tabular}{|l|c|c|c|}
\hline Circuit & Type & DC Poles & $\begin{array}{c}\text { Series/Parallel } \\
\text { PSS }\end{array}$ \\
\cline { 1 - 2 } TF & I & 2 & $1 / 4$ \\
\cline { 1 - 2 } OH & III & 2 & $6 / 2$ \\
\hline PF1a Upper & II & 3 & $2 / 1$ \\
\cline { 1 - 1 } PF1a Lower & & & $2 / 1$ \\
\cline { 1 - 2 } PF1b & I & 2 & $2 / 1$ \\
\hline PF2 Upper & II & 3 & $2 / 1$ \\
\cline { 1 - 1 } PF2 Lower & & & $2 / 1$ \\
\hline PF3 Upper & IV & 3 & $2 / 1$ \\
\cline { 1 - 2 } PF3 Lower & & & $2 / 1$ \\
\hline PF4/5 & I & 2 & $2 / 1$ \\
\hline CHI & I & 2 & $2 / 2$ \\
\hline Spare & & 2 & 2 \\
\hline Total & 12 ckt & 21 pole & 42 PSS \\
\hline
\end{tabular}

The voltage applied to, or the current produced in, each of the circuits must be explicitly controlled. Therefore, eleven (11) separate sub-circuit control loops are required in case all of the bus links are in place, plus one spare, for a total of twelve (12). However, it is noted that if the bus links are not installed in the Type II and Type IV circuits, then they reduce to Type I and Type III, respectively, and the number of explicit control loops is correspondingly reduced. When the bus links are not in place two halves of the former Type II or Type IV circuits are treated in all respects by the PSRTC as single Type I or Type III circuits, respectively. Currents in twenty one (21) poles are measured and monitored.

\section{POWER SUPPLY REAL TIME CONTROLLER}

\section{A. Power Supply Control Function}

The Power Supply Control Function is depicted in Figure 4. The same basic function is applied to all of the circuits. The output of the control function consists of the firing angle $(\alpha)$ and convert bit commands which are delivered to the PS in the form of the PS Control Words. Since the firing angle is related to the voltage, these commands are, essentially, voltage commands. The voltage commands are derived either from closed loop proportional - integral (PI) current control against a programmable reference, or from a direct voltage request. The direct voltage request is obtained from either a programmable reference, or a signal supplied in real time by the PRTC. When in the voltage control mode, a current clamp feature is applied if the current approaches a programmable limit. Additional features include rate limiting, and control of antiparallel rectifiers, including the required $\alpha$ limiting.
Following is a description of these features, with reference to Figure 4.

\section{1) Closed Loop Current Control}

The load current $I_{l o a d}$ is subtracted from a programmable

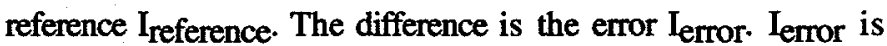
multiplied by proportional gain $G_{p}$, and is integrated and multiplied by integral gain $\mathrm{G}_{\mathbf{i}}$. The sum of these two terms is the voltage requested by current control $V_{\text {current. The }}$ integrator includes an "anti-wind-up" reset feature which is a function of time; one reset time shall be allowed per circuit.

\section{2) Voltage Control \& Current Clamp}

The source of the command $V_{c o m m a n d}$ can be either the programmable reference $V_{\text {reference }}$ or a real time signal provided by the PRTC VPRTC. The time window during which VPRTC is enabled shall correspond to the PCW. Voltage control is subject to an override by a current clamping feature which comes into play when limits are reached. The clamping is based on PI control which generates a voltage adjustment term $V_{\text {clamp }}$ whenever the load current $I_{\text {load }}$ passes outside the range between the positive and negative clamp settings $\mathrm{I}_{\text {clamp }}>0$ and $\mathrm{I}_{\text {clamp }}<0$. When the current returns to the allowable range the clamp integrator is reset. The net voltage request based on voltage control $V_{\text {voltage }}$ is the result of the addition of $\mathrm{V}_{\text {command }}$ and $\mathrm{V}_{\text {clamp }}$.

\section{3) Voltage to Alpha Conversion}

The selection of the source of the voltage request $V_{0}$ from either voltage control $V_{\text {voltage }}$ or current control $V_{\text {current }}$ is a function of time. $V_{1}$ is the per unit version of $V_{0}$ based on $\mathrm{V}_{1}=\mathrm{V}_{0} / \mathrm{V}_{\max }$, where $\mathrm{V}_{\max }$ is the maximum no-load voltage available for the circuit in question. $V_{\max }$ is based on the programmable number of series PSS (NS), and the primary side $\mathrm{AC}$ rms line-to-line source voltage $\mathrm{E}$ (nominally $13.8 \mathrm{kV}$ at $D$-site). $V_{2}$ is a rate and magnitude limited version of $V_{1}$, where the rate limit is programmable for each circuit and the magnitude limit is -1 to 1 per unit. The rate limit is only applied when the antiparallel mode is in effect (see next section). $\alpha_{0}$ is the firing angle request, and $\alpha_{1}$ is the final firing angle command after limiting. If antiparallel operation is not in effect, then the allowable range of $\alpha_{1}$ is 0 to $\alpha_{\max }$, where $\alpha_{\max }$ is the maximum permissible delay angle. It is calculated based on the load current per PSS, equal to $\mathrm{I}_{\text {load }} / \mathrm{NP}$ where NP is the number of parallel PSS branches in the circuit being controlled, the $\mathrm{AC}$ source frequency $\omega$, the commutating inductance $\mathrm{L}_{\mathrm{c}}$, and the $\mathrm{AC}$ source voltage $\mathrm{E}$, along with a programmable inversion margin angle $\gamma$. If antiparallel operation is in effect, then the minimum permissible delay angle $\alpha_{\min }$ is set equal to $180-\alpha_{\max }$, so that the allowable range of alpha is therefore $\alpha_{\min } \leq \alpha_{1} \leq$ $\alpha_{\max }$ in the antiparallel mode. 


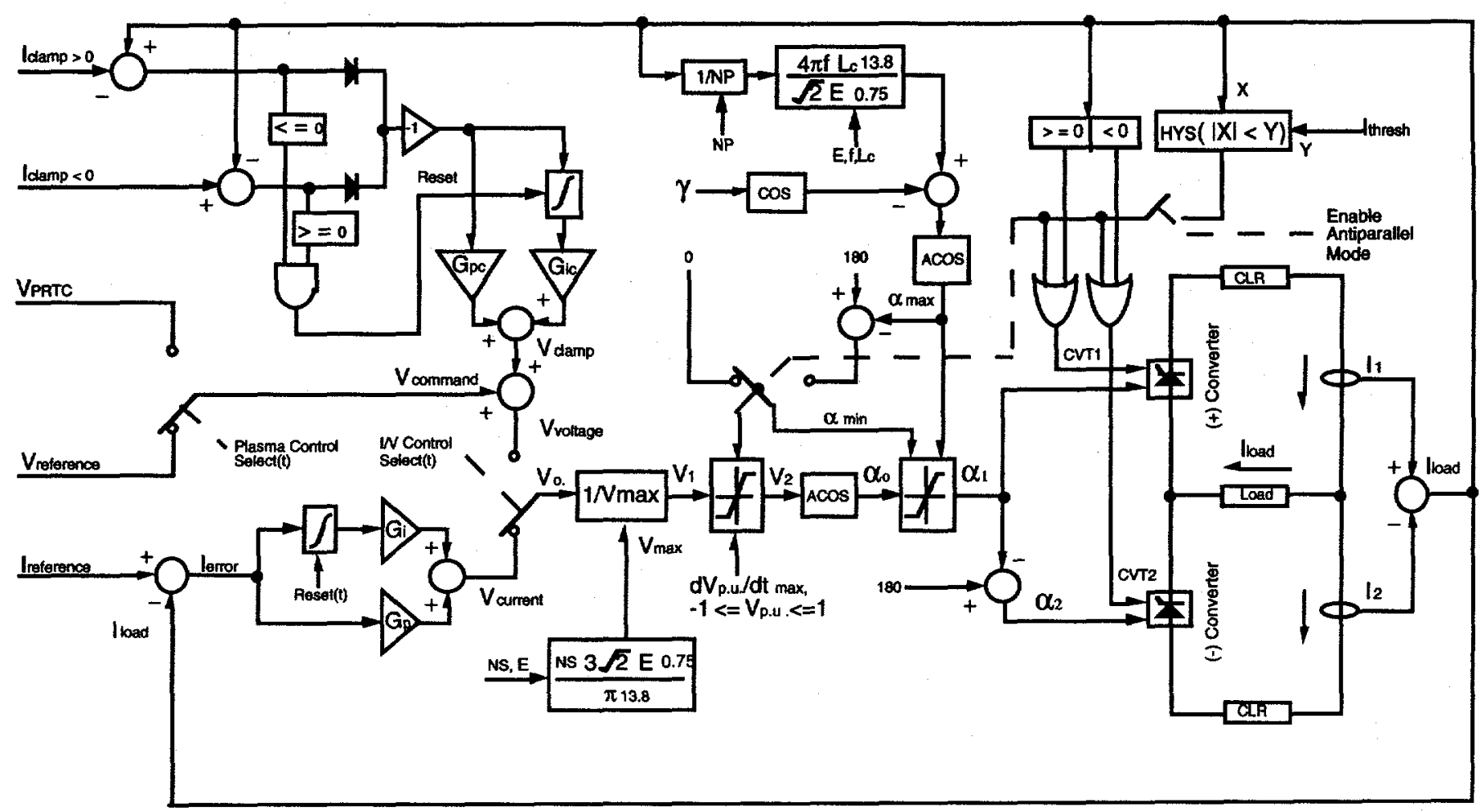

Figure 4: Power Supply Control Function

\section{4) Antiparallel Operation}

Antiparallel Operation permits bipolar current flow in the load without delay around current zero. When it is in effect, the antiparallel converters (a.k.a. the $(+)$ and $(-)$ converters) must be operated at symmetric (about 90 degrees) delay angles $\alpha_{\min }$ and $\alpha_{\max }$. This is required to minimize the circulating current which is impeded only by the Current Limiting Reactors (CLRs). The antiparallel mode is declared when the magnitude of the load current $\mathrm{I}_{\text {load }}$ falls below a threshold $\mathrm{I}_{\text {thresh. }}$. Thus the comparison is conditioned by a hysteresis function which only declares the mode to be true if the previous magnitude exceeded the threshold. A switch is provided to enable/disable antiparallel operation for each circuit.

\section{B. Current Measurements}

PSS branch currents are measured by several types of DC currrent transducers; redundant measurements are provided in each branch. At each time step, the difference between the magnitude of the two measurements is calculated (after applying scale factor including polarity) and compared to a programmable allowable as a check for the validity of the measurements. The measurement with the larger magnitude is used for further processing. The coil currents $I_{\text {load }}$ are obtained from the sum of the individual branch measurements.

\section{Protection Features}

Following protection features are provided in the PSRTC:

- Instantaneous overcurrent protection

- single pulse fi2(t)dt protection
- adiabatic coil temperature rise protection

- RMS current checks (based on continuous simulation of heating (during pulse) and cooling (between pulses) of DC cable systems.

\section{Simulation Mode}

In order to test the PSRTC, a simulation package is included which interfaces with the real time control code at the same points of input and output as in normal operation (the inputs being the PSS branch currents and the outputs being the $\alpha$ commands to the power supplies. Following features are included:

- Transient solution of multiple mutually coupled $L / R$ circuits (10 simulation time steps per control system time step) using PPPL's' LRSIM code

- Simulation of latency of input data by utilization of simulation results obtained midway between control system time steps as inputs to control algorithm

- Simulation of transient heating of coils and its effect on ohmic resistance

- Simulation of delays and limits of power supplies

\section{E. Implementation}

The PSRTC is written primarily in FORTRAN (some adjuncts are in C) and runs on a VME based computer system inherited from prior experiments at PPPL [4]. It consists of the following components: 
- Themis host computer (Sparc 10 running Sun OS)

- ethernet connectivity

- user interface

- data management

- $\quad$ software development

- Skybolt I Shamrock real time computer

- four parallel Intel 80860 processors

- 64MB shared memory

- DMA port

- Precision Filters data acquisition system

- two 32 channel analog input modules

- 10kHz

- DMA to Skybolt

- Power Conversion (PC) Link driver

- interface to serial command link which distributes commands to 39 distributed PS at PPPL facility

The PSRTC occupies one of the four parallel i860 processors; the other three are available to the PRTC. The control system update rate is $1 \mathrm{kHz}$ (once per millisecond). Typical execution time of the PSRTC algorithms with all circuits running is less than $800 \mu \mathrm{S}$.

The PSRTC utilizes 47 of the 64 DAS channels available. The remainder are reserved for the PRTC Ip, flux loop, and B probe signal inputs. These signals are transmitted from the transducers located in the NSTX test cell to the location of the control computer rack via analog fiber optic links.

\section{F. Future Plans}

A graphical user interface is under development to provide capabilty for editing the text files used to input the control and waveform data.

An upgrade to a new computer system [3] is planned for late 1999 (a.k.a. "day 2"). This will include:

- Upgraded Themis host computer

- Upgraded Sky computer

- Distributed data acquisition system based on multiple VME crates strung together using fiber optic links and Front Panel Data Port (FPDP) direct access to the real time processor.

The new computer system is not requined in principle for the PSRTC but will instead facilitate an advance in the PRTC development, including more than a doubling of the number of inputs.

\section{REFERENCES}

[1] C. Neumeyer, et al, "Engineering Overview of the National Spherical Torus Experiment", Transactions of $17^{\text {th }}$ IEEE/NPSS Symposium on Fusion Engineering, San Diego, CA, October 1997, IEEE No. 0-7803-4226-7/98

[2] D. Gates, et al, "NSTX Control System Development Plan", RT99 Paper \#181

[3] D. Mueller, et al, "Upgrade to NSTX Control Computer", RT99 Paper \#144

[4] P. Sichta et al, "Computing Hardware for the PBX-M Plasma Control System, ", Transactions of $16^{\mathrm{h}}$ IEEE/NPSS Symposium on Fusion Engineering, Champaign, Ill, October 1995, IEEE No. 0-7803-2969$4 / 95$ 